\title{
Proceedings of the meeting of the Association of British Neurologists held jointly with the Polish Neurological Society in London, April 1984.
}

\author{
CLUSTER HEADACHE AND ERYTHROCYTE \\ CHOLINE LEVELS \\ J de Belleroche, GE Cook, I Das, R Joseph \\ and $\mathrm{F}$ Clifford Rose. Charing Cross Hospi- \\ tal, London.
}

Erythrocyte choline levels are known to be significantly altered in manic depressive disease, a condition which responds well to lithium treatment. We have measured erythrocyte choline levels in cluster headache patients and age related control subjects since lithium is also beneficial in this condition. Choline levels were found to be significantly reduced in cluster headache patients both during a cluster period (nine subjects) and between clusters (10 subjects), being $58 \%$ and $55 \%$ of the control level $(4.73 \pm 0.31 \mu \mathrm{mol} / \mathrm{l}$ choline: mean value $\pm S E M$ for 14 subjects) respectively. Choline levels in cluster headache patients (eight subjects) were greatly elevated by lithium treatment rising to 78 times the control level after 2 weeks treatment (11.2 $\mathrm{g}$ lithium carbonate). The presence of depressed choline levels both during and between cluster attacks indicates that this may be a predisposing condition which results in a cluster attack only when associated with a trigger factor. These results were compared with those obtained from patients with classical and common migraine and with tension headache

THE ROLE OF PROGRAMMED CELL DEATH IN THE DEVELOPMENT OF THE EYE

CL Scholtz. The London Hospital, London.

There are three steps at which programmed cell death is important in the development of the eye; firstly the absorption of mesenchyme as the optic vesicle approaches the surface ectoderm, secondly in the closure of the optic fissure and finally in the ganglion cell layer of the retina. In this study a complicated colobomatous microphthalmia inherited in the mouse was used as a model to examine these three stages in the development of the eye. Serial sections of embryos from day ten to adult life showed that the lens formed normally and that the mesenchyme was resorbed.
Failure of cell death in the walls of the optic fissure resulted in the coloboma. Excessive ganglion cell death was observed in the retina probably because the axons of these cells failed to synapse with other visual areas. It is proposed that failure of cell death is the major cause of the abnormal development.

\section{CLINICAL MANIFESTATIONS OF HEREDOPATHIA ATACTICA \\ POLYNEURITIFORMIS}

FB Gibberd and JM Goldman. Westminster Hospital, London.

A study of six patients with Refsum's disease demonstrated that the clinical abnormalities fall into three categories. Although failure to metabolise phytanic acid is diagnostic, not all the manifestations can be explained by phytanic acid intoxication.

Group 1 Congenital abnormalities. Skeletal abnormalities, not present in our cases, are the commonest of these reported in the literature. Anosmia may fall into this category.

Group 2 Chronic slowly developing signs. Retinitis pigmentosa is the best known of this group. Small pupils, which do not dilate, and cataracts limit vision, especially in the dark.

Group 3 Acute relapsing symptoms. The most sudden is a cardiac arrhythmia, which may lead to death. Neuropathy comes on more slowly and recovers slowly when the plasma phytanic acid falls. This category of symptoms is often associated with intercurrent illness, especially if it is associated with anorexia. The fatty acids, mobilised from the adipose tissue, are metabolised except for phytanic acid which rises in concentration and leads to an exacerbation of the Group 3 symptoms.

SECONDARY MUSCLE CARNITINE DEFICIENCY DUE TO A NEW INBORN ERROR OF

METABOLISM

DM Turnbull, DL Stevens, K Bartlett, HSA Sherratt. Gloucestershire Royal Hospital, Gloucester, and University of Newcastle upon Tyne.

A 53 year-old-woman presented 7 years ago with proximal muscle weakness. Exe tion increased her symptoms and a sho rest allowed some return of muscte strength. Muscle biopsy showed exce每 neutral lipid especially in Type I fibre There was a low concentration of carnitine in muscle and serum. Treatment with ora $\mathrm{D}, \mathrm{L}$-carnitine was started $(2-9 \mathrm{~g}$ per days in combination with varying doses of pred. nisolone. Fluctuations in the patient's clin? cal condition occurred which were unr $\vec{e}$ lated to treatment. Further investigation were performed because of a modest bu definite deterioration in skeletal muscte function. A repeat muscle biops confirmed excess lipid accumulation and low free carnitine concentration. The rate? of oxidation of fatty acid derivatios $\overrightarrow{e s}$ (palmitoyl-carnitine, palmitoyl-CoA बె! octanoate) by skeletal muscle mitoc䍐古 drial fractions was only $20 \%$ of copri rates. A defect of the short-chain acyl- 0 dehydrogenase (an enzyme of $\beta$-oxide्wion of fatty acids) was confirmed by dide assay. Impaired metabolism of the storty chain acyl-CoA was also indicated by excretion of large amounts of ethylrifio nate. Carnitine deficiency appears t $\vec{\sigma} \overrightarrow{b g}$ secondary when there is incomplete oxidg tion of fatty acids. Short-chain acyl-Co A dehydrogenase contains flavin prostheta groups, and riboflavin therapy (1) $\mathrm{mg} /$ day) produced a considerabf improvement in muscle strength. Carnition deficiency is probably a secondary phenomenon and not a primary defect $\frac{\mathrm{O}}{\mathrm{m}}$ most cases of lipid storage myopathy.

ORAL 3,4-DIAMINOPYRIDINE IN THE TREATMENT OF THE LAMBERT-EATON MYASTHENIC SYNDROME (LEMS)

NMF Murray, J Newsom-Davis, Y Kar CM Wiles. National Hospital for Nervous Diseases, London.

Nerve-impulse evoked transmitter releaß̧ at the motor nerve terminal is reduced the LEMS. The aminopyridines increase acetylcholine release by blocking $\mathrm{K}^{+}$conductance, thereby prolonging the action potential and increasing voltage-depende $\mathrm{Ca}^{2+}$ influx. Blood-brain barrier penetrof tion by 3,4-diaminopyridine $(3,4-\mathrm{DAP})$ is 
less than by 4-aminopyridine (4-AP) which, although effective in increasing transmitter release, causes prominent central nervous system side effects. We have assessed the effectiveness of 3,4-DAP in eight LEMS patients. Evaluation included a subjective analogue scale of symptoms, a timed walking test, force recordings and measurements of the amplitude of the evoked muscle action potential during repetitive nerve stimulation. A double blind comparison was made between a single oral dose of $20 \mathrm{mg} \mathrm{3,4-DAP}$ and $120 \mathrm{mg}$ pyridostigmine in six patients. Improvement was more marked with 3,4-DAP in five by both clinical and electrophysiological criteria. The peak effect occurred 1-2 hours after administration. With chronic 3,4-DAP treatment (30-90) $\mathrm{mg} /$ day in divided doses for up to 9 months: $\mathbf{n}=8$ ) there was a clear cut improvement in four and more marginal benefit in the remainder. Circumoral and peripheral paraesthesiae were frequent but not troublesome. No fits or confusional states occurred. We conclude that 3,4DAP is associated with fewer side effects than 4-AP and is clinically as effective. This preparation may be useful in the treatment of the Lambert-Eaton myasthenic syndrome.

\section{MOTOR NEURON DISEASE--THE PRACTICAL} PROBLEMS

PG Newrick, R Langton Hewer. Frenchay Hospital, Bristol

Experience indicates that the management of motor neuron disease (MND) is frequently not satisfactory. In order to provide an improved service it is necessary to have knowledge of the problems involved. Unfortunately, the literature does not provide clear guidance. We describe a factual study. Forty-two patients with definite MND, from the Departments of Neurology at Bristol and Southampton, were interviewed by the same doctor. Information was obtained on disease duration, the level of disability, symptoms, housing, and the use of aids. Disability was scored on a new scale $(0-25)$ which assesses bulbar function, limb function. and sources of patient care. Of the 42 patients, 20 judged themselves as independent, and eight as totally dependent. This correlated with their disability score -18 of the 20 independent patients scoring $>20 / 25$ and all eight totally dependent patients scoring $<10 / 25$. Pain, falls, constipation, leg swelling and sleep disturbance emerged as major symptoms. Twenty-four per cent of patients appeared to be in need of an aid (for example, walking frame) and in a further $49 \%$ of cases the aid was provided unsatisfactorily late in the course of the disease. More than half said that they disliked attending neurology outpatient clinics. Criticism centred around poor transport arrangements, lack of information about symptom control. and unsatisfactory help from junior staff. We suggest that the management of motor neuron disease requires urgent review.

REGIONAL CEREBRAL OXYGEN UTILISATION. CEREBRAL BLOOD FLOW. AND

BLOOD-BRAIN-BARRIER INTEGRITY IN MULTIPLE SCLEROSIS

DJ Brooks, KL Leenders, J Marshall, NJ Legg, T Jones. Hammersmith Hospital, London

Using steady-state inhalation of $\mathrm{C}^{15} \mathrm{O}_{2}$ and ${ }^{15} \mathrm{O}$, combined with positron emission tomography (PET), regional cerebral oxygen utilisation $\left(\mathrm{rCMRO}_{2}\right)$ and cerebral blood flow ( $\mathrm{rCBF}$ ) have been measured in fifteen patients with multiple sclerosis (MS). A significant reduction in oxygen utilisation by both central white and peripheral cortical grey matter was found for the MS group-compared to a group of normal controls. This reduction in $\mathrm{rCMRO}_{2}$ was accompanied by a coupled reduction in blood flow and correlated well with the presence of atrophy on X-ray CT scans. No apparent correlation between $\mathrm{rCMRO}_{2}$ and locomotor dysfunction or clinical disease duration was found. The integrity of the blood-brain-barrier (BBB) was assessed using ${ }^{82} \mathrm{Rb}^{+}$, a positronemitting analogue of $\mathrm{K}^{+}$and PET. In normal cerebral tissue the $\mathrm{BBB}$ is highly impermeable to $\mathrm{K}^{+}$ions. We found no significant difference in $\mathrm{Rb}$ extraction between the MS group and normal controls suggesting that demyelination does not lead to a breakdown in BBB integrity. In conclusion PET provides a sensitive method of assessing cerebral hypofunction in multiple sclerosis. Profound generalised hypofunction can be present in MS patients with only slight clinical disability.

\section{SCREENING OF CSF ANTIBODY}

SPECIFICATIONS IN MULTIPLE SCLEROSIS

Brenda Belshaw, Ingrid $V$ Allen, DFW McCormick, GB Wisdom, DR Hawkins. The Queen's University, Belfast

The predominant immunological change observed in multiple sclerosis is the elevation of immunoglobulin levels in the CSF.
The aim of this work was to determine the specificity of some of the CSF antibodies and, in particular, to look for indications of autoimmune responses. An enzyme-linked immunosorbent assay using nitrocellulose membrane discs as solid phase was developed for the detection of CSF antibodies. Using the assay 18 multiple sclerosis CSFs, 10 CSFs from patients with other neurological disorders and $20 \mathrm{CSFs}$ from orthopaedic patients were screened for autoantibodies to extracts from brain, lymphoid and other tissues and to some viruses. The levels of antibodies to the tissue extracts were found to be the same in multiple sclerosis patients and other patients. However, multiple sclerosis CSFs tended to have somewhat stronger responses to viral antigens, in particular, mumps and measles. It would appear that autoantibodies to the tissues tested are not a feature of multiple sclerosis and nonspecific immuno-stimulation may be responsible for the increased levels of CSF viral antibodies.

\section{CELLULAR SENSITIVITY TO IONISING} RADIATION IN FRIEDREICHS ATAXIA PD Lewis. Hammersmith Hospital, London

A mild increase in cellular sensitivity to ionising radiation. as shown by impaired post-irradiation clonal growth of fibroblasts, has been demonstrated in Friedreich's ataxia. Susceptibility of chromosomes from patients with Friedreich's ataxia to changes induced by ionising radiation and chemical mutagens is increased. Pilot studies suggested that in this disease there is also impairment of cellular reparative mechanisms after irradiation, and the occurrence of alterations in newly synthesised DNA. The data currently available are consonant with the view that Friedreich's ataxia is a disorder in which cellular sensitivity to DNA-damaging agents is abnormally high. The relationship of such hypersensitivity to the degenerative neurological abnormalities that characterise this disease remains a matter of speculation.

MICROVASCULAR DECOMPRESSION: DEFINITIVE SURGICAL TREATMENT WITHOUT NERVE SECTION FOR TRIGEMINAL AND GLOSSOPHARYNGEAL NEURALGIAS

R Illingworth, P Richards. Charing Cross Hospital, London

For over 25 years it has been known that patients with trigeminal neuralgia (TN) 
and glossopharyngeal neuralgia (GPN) have anatomical and pathological abnormalities at the root entry zones of the Vth and IXth nerves. Between July 1976 and December 198339 patients, ages 30-80 years (mean 57.4), with TN and GPN have been treated by posterior fossa microvascular decompression. Cross compression of the nerves by arteries was found in 33 (combined with veins in five), by veins alone in four, and by tumours in two. Decompression of the nerves relieved the pain in all patients but two have relapsed at 6 months. No sensory loss was produced but three patients who had no previous section have noticed minor paraesthesiae. One patient had a delayed and temporary VIIth palsy, there was one delayed but permanent ipsilateral deafness, and one temporary IVth palsy, follow-up extends to 5 years, (mean 27 months). The procedure appears to be a safe and effective method of relieving TN and GPN without producing sensory loss. It is well tolerated even in older patients ( six were over 70 years old), and should be considered as the primary surgical procedure when medical management fails.

PERINEAL, PUDENDAL AND TRANSCUTANEOUS SPINAL STIMULATION; NEW METHODS FOR INVESTIGATION OF URINARY AND FAECAL INCONTINENCE

S Snooks, M Swash. St Mark's Hospital and the London Hospital, London

We have used two new methods, digitally directed pudendal nerve stimulation and trancutaneous spinal stimulation to measure the latency from the point of stimulation to the evoked muscle action potential (MUAP) in the striated urethral sphincter musculature. These methods have been developed from those applied to investigation of the external anal sphincter muscle in patients with faecal incontinence. In 20 normal subjects the perineal nerve terminal latency following pudendal nerve stimulation, using an indwelling intraurethral surface electrode mounted on a catheter for recording, was $2.4 \pm 0.2 \mathrm{~ms}$. In a group of 20 patients with "idiopathic" double incontinence, the perineal terminal nerve latency was $4.3 \pm 1.6 \mathrm{~ms}(\mathrm{p}<0.01)$. In the seventeen patients with double incontinence the spinal nerve terminal latency to the urethral striated sphincter muscle was $6.9 \pm 1.8 \mathrm{~ms}$ from $\mathrm{L} 1$ and 5.5 $\pm 1.6 \mathrm{~ms}$ from L4. In normal subjects the latency to the periurethral striated muscles was $4 \cdot 85 \pm 0 \cdot 14 \mathrm{~ms}$ from $\mathrm{Ll}$ and $4.06 \pm$
$0 \cdot 1 \mathrm{~ms}$ from $\mathrm{L} 4$. These results indicate damage to the distal part of the innervation of the urethral sphincter musculature in double incontinence. This distal nerve lesion is similar to that which we have previously reported in the pudendal innervation of the external anal sphincter in patients with faecal incontinence.

ORGANISATION OF SUBSTANTIA NIGRA PROJECTIONS TO THE THALAMUS, TECTUM, AND RETICULAR FORMATION

J Mitchell, PN Leigh, K Lyons. Wessex Neurological Centre, Southampton

We have studied the organisation of efferent projections from the rat substantia nigra (SN) using a retrograde doublelabelling technique to examine collateral arborisations. The fluorescent dyes True Blue (TB) and Nuclear Yellow (NY) were injected stereotactically into the thalamus, superior colliculus or brain stem reticular formation. Single and double labelled SN neurons were detected under fluorescence microscopy, charted onto diagrams of SN and counted to give quantitative data on the degree of arborisation. Retrogradely labelled neurons within $\mathrm{SN}$, pars compacta, were seen after injection of dyes into the caudal midbrain, superior colliculus, angular complex (medial midbrain reticular formation) and thalamus. Double-labelled neurons were seen in the ventro-medial and ventrolateral SNR after injection into superior colliculus and angular complex; single labelled neurons (projecting to angular complex but not to superior colliculus) were seen in the dorsal SNR. However, this dorsal group of neurons projected to ventromedial and parafascicular thalamic nuclei; combined thalamic and angular complex injections resulted in double-labelled neurons in dorsal SNR. Our findings suggest a major nigral projection to the angular complex which can be differentiated anatomically from the nigro-collicular projection. The possible role of these nigral outputs in motor behaviour will be discussed.

THE DRUG TREATMENT OF SEVERE GENERALISED OR AXIAL PRIMARY TORSION DYSTONIA

CD Marsden, N Quinn, Marie-Helene Marion. Institute of Psychiatry, London

The drug treatment of dystonia is difficult and unpredictable. We describe our use of high-dose anticholinergic therapy to control severe generalised dystonia in children, and of combined tetrabenazine pimozide/anticholinergic drug treatment for disabling axial dystonia in adults. Ben zhexol (up to $30 \mathrm{mg}$ for 6 months) proved superior to placebo in six young patient\$s Subsequently, we have employed higher doses ( 60 to $120 \mathrm{mg}$ daily) in a further $1 \notin$. patients with even greater benefit (judge by a standardised dystonia rating scale): Anticholinergic toxicity was reduced $b \overline{\overline{5}}$ increasing the dose gradually over months Learning and memory were not obviousl impaired. Anticholinergics were of les: value in adults, who developed more toxic ity at lower doses than chiddren. Eleve adults unable to sit or walk because of severe dystonia of the trunk were restored to independence by a fixed low dose ( $\mathrm{t} \Phi$ avoid depression) of tetrabenazine ( $25 \mathrm{mg}$ tds), to which was added pimozide ( $2 \mathrm{mg}$ mane, gradually increasing by 1-2 m increments) until dystonia was controlle or disabling drug-induced Parkinsonism appeared, at which point benzhexol of orphenadrine was added. The dose of pimozide, and of the anticholinergic, wer then titrated to achieve the best controb of the dystonia with the minimum Parkinsono ism.

PROGNOSIS IN AMAUROSIS FUGAX

GS Shaw. Royal Victoria Infirmary, Newc解居 upon Tyne

The frequency and pattern of visual loss im amaurosis fugax have been analysed from hospital case records from 152 patien seen between 1966 and 1982. Fifty-two per cent of patients had had fewer than te attacks at the time of initial presentation In $53 \%$ of patients, visual loss durin attacks was complete; in $32 \%$ it was par tial, and in $14 \%$ both complete and partio attacks occurred. Cerebral infarction antedated or coincided with the onset amaurotic attacks in $5 \%$ of patients, and $18 \%$ had previous or coincident ipsilater hemispere transient ischaemic attacks. Five्s per cent suffered a complete stroke in thio 12 months following hospital presentatio and thereafter the incidence of stroke fetb to $1 \%$ per annum. Prognosis varied with the frequency and pattern of visual loss, and the outcome was best in those wit fewest attacks and in those whose attacks were likened to a curtain descending from above. Details of other prognostic factors and of the outcome for patients in the cohort will be presented. 
CEREBRAL BLOOD FLOW AND BLOOD VOLUME IN OCCLUSIVE CAROTID ARTERY DISEASE: CIRCULATORY RESERVE BEFORE AND AFTER EC-IC BYPASS

JM Gibbs, RJS Wise, RJS Frackowiak, NJ

Legg. Hammersmith Hospital, London

The role of intracranial-extracranial (ECIC) bypass surgery remains undefined, but continuing TIAs distal to an occluded carotid artery may justify the procedure in some cases. We have studied such patients in an attempt to identify those most likely to benefit from bypass surgery. Regional cerebral blood flow (CBF), blood volume (CBV) and fractional oxygen extraction (OER) were measured by positron emission tomography in 30 patients with complete internal carotid artery occlusion. In the majority of cases, any reduction of CBF distal to an occluded carotid artery was matched to diminished cerebral oxygen demands. CBF was inappropriately low in only a small minority of patients, in whom cerebral oxygen metabolism was maintained by a compensatory rise of OER. The frequent finding of increased CBV distal to occluded vessels was consistent with a state of focal vasodilatation in response to diminished cerebral perfusion pressure. Analysis of the relationship between CBF, CBV and OER suggested that the degree of reduction of perfusion pressure, and hence circulatory reserve, could be most reliably predicted by the ratio $\mathrm{CBF} / \mathrm{CBV}$. In 12 patients re-studied after EC-IC bypass surgery there was no change of $\mathrm{CBF}$ but a significant post-operative fall of $\mathrm{CBV}$, implying that cerebral perfusion pressure had been increased by the procedure. By identifying those patients with carotid occlusion who are most compromised on haemodynamic grounds, combined measurement of CBF and CBV should be of value in selection of candidates for EC-IC bypass surgery.

YOUNG STROKE-A SURPRISING ASSOCIATION WITH TRAUMA

D Hilton-Jones. The Radcliffe Infirmary, Oxford

In a retrospective study of the five years 1978-1982 80 patients under the age of 45 years were identified as having presented with stroke (excluding aneurysmal subarachnoid haemorrhage) to one of the Neuroscience Departments of the Radcliffe Infirmary, Oxford. The purpose of studying this group of patients was to determine any common predisposing factors to young stroke and to assess the value of specific investigations including cardiac evaluation and cerebral angiography. Twenty patients had suffered intracranial haemorrhage, leaving 60 with presumed or proven hemisphere or brain stem infarction. In this latter group the single commonest identifiable predisposing factor to stroke was trauma, which occurred in 13 of the 60 patients. A variety of systemic disorders were identified but their relationship to the stroke was not always clear. Detailed cardiac investigation and cerebral angiography were rarely of help in determining aetiology or in aiding management.

\section{THE NEUROLOGY OF CHRONIC HYPOXIA} C Clarke. St Bartholomews Hospital, London

Chronic hypoxia with hypocapnia is experienced by high altitude climbers and trekkers. Acute Mountain Sickness (AMS) is a common direct effect-a self limiting illness of headache, vomiting and intense malaise. AMS may progress to more serious conditions-High Altitude Pulmonary Oedema and High Altitude Cerebral Oedema (HACO). This paper summarises the clinical findings in HACO. The condition occurs in two situations-during the acclimatisation process at altitudes between 4000 and 5000 metres where the ambient $\mathrm{pO}_{2}$ is between 70 and $80 \mathrm{mmHg}$ and at extreme altitude, above 7000 metres, where the ambient $\mathrm{pO}_{2}$ is less than $60 \mathrm{mmHg}$. Patients become sleepy, irrational and confused. There are a wide variety of early neurological signs the most prominent being disturbance of behaviour, truncal ataxia and papilloedema. The condition is frequently fatal. Haemorrhagic infarction of the brain is found at necropsy. The pathophysiology of HACO is believed to be related to the marked changes in cerebral blood flow which increases by $25 \%$ on acute exposure to moderate altitudes eg 3500 metres. Vasogenic cerebral oedema follows. The recognition of this important neurological syndrome has added to the understanding of the effects of hypoxia and is of practical value to the many thousand native lowlanders who now travel annually to the mountain regions of the world.

\section{PROBABLE MECHANISMS OF VALPROATE} HEPATOTOXICITY

DJ Dick, HSA Sherratt, DM Turnbull. University of Newcastle upon Tyne, Newcastle upon Tyne

Valproate is an important anticonvulsant which has been associated with hepatotoxicity. This adverse effect is of considerable concern since it is often sudden in onset, and irreversible. The hepatic pathological changes consist predominantly of fatty infiltration and necrosis. Valproate is a branched chain fatty acid which inhibits fatty acid oxidation, pyruvate oxidation, gluconeogenesis and urea synthesis in rat liver in vitro and also causes hypoketonaemia and hypoglycaemia in fasted rats. The effect of valproate on intermediary metabolism in humans was therefore studied. Oral administration of valproate $(1 \mathrm{~g})$ to fasted (for $18 \mathrm{~h}$ ) male subjects caused a fall in blood glucose concentration and increase in the blood concentration of gluconeogenic precursors. The most marked effect of valproate administration was on ketone bodies (a $77.5 \%$ fall in 3-hydroxybutyrate and a $37.5 \%$ fall in acetoacetate concentrations). Similar changes in the blood concentration of intermediary metabolites were observed after the administration of $400 \mathrm{mg}$ of valproate intravenously. This study clearly demonstrates that valproate has marked effects on intermediary metabolism in man and suggests that the metabolic inhibition seen in the rat also occurs in man. Although the inhibitions caused by therapeutic concentrations of valproate are partial and well tolerated, they could significantly impair liver function if there is an additional problem, such as an inborn error of metabolism or when patients are on multiple drug therapy.

PERFOMANCE SCORE SYSTEM FOR MONITORING SEVERE NEUROMUSCULAR DISEASE

Y Karni, K Mills, CM Wiles. The National Hospital for Nervous Diseases, London

Increasing availability of potential treatments for peripheral neuromuscular diseases requires that adequate means are used for monitoring clinical progress. In illness dominated by muscle weakness strength measurements ${ }^{1}$ are of value but the need was felt for a reproducible set of perfomance tests. Twenty simple tests were designed to span disability ranging from "bed bound and ventilated" to "able to run". Three timed elements (hours per day on ventilator, time to walk $\mathbf{3 0}$ metres, time to go up and down 10 stairs) were incorporated but the score elements all required YES/NO answeres. Each full assessment takes about 15 minutes and the results are stored on floppy disc or recorded on a simple annotated score sheet. The assessment 
has been evaluated in $\mathbf{7 0}$ patients for a follow up period of up to 2 years. Interobserver errors were measured by asking five examiners each to test six patients: the majority of the small differences found were caused by incorrect application of the test and only occasionally was a practice effect seen. The system provides a reliable method of recording major changes in performance and, when linked with strength measurements, permits a more comprehensive view of the patient's progress over prolonged periods of time.

\section{Reference}

' Wiles CM. Karni Y. J Neurol Neurosurg Psychiatry 1983;46:1006-13.
The following papers were presented by the members of the Polish Neurological Society:

ENDOC RINOLOGICAL ASPEC 'TS OF EPILEPSY D. Rosciszewska

BIOAVAILABILITY OF TWO TYPES OH

CARBAMAZEPINE TABLFTS

W Kuran, K Niedzielska

OPHTHALMOLOGICAL AND VEP FINDINGS IN SSPF:

W Horyd, W Sobczak

NEUROLOGY IN POLANI): SERVI(E ANI) RESEARCH

I Wald

SUBACUTE SCLEROSING PANENCEPHALITIS WITH IMPROVEMENI

J Kulczycki

ACTH TRE:ATMENT IN MYASTHENIA GRAVIS

E Manko

GENETICS OF THE CHRONIC PROXIMAL FORM OF SPINAL MUSCÚLAR ATROPHY

I Hausmanowa-Petrusewicz, J Zaremba
DIAGNOSTIC YIELD OF EMG AND BIOPSY IN SPINAL MUSCULAR ATROPHY

I Hausmanowa-Petrusewicz FFFFCI OF PREDNISONF MEDICATION ON ANTI-MAG. ANTI-MBP FIBRE ANTIBODIES AND CIC LEVEL IN CSF IN MULTIPLE SCLEROSIS A Wajgt. M Gorny EFFECT OF IMMUNOSUPPRESSIVE DRUGS ON INTRATHECAL IgG PRODUCTION IN MULTIPII SCLEROSIS

$M$ Wender, A Wajgt

DIAGNOSTIC SIGNIFICANCE (OF EVOKED)

POTENTIALS IN MULTIPLE SCLEROSIS

T Domzal, J Kotowicz. DIAGNOSTIC VALUE OF AUDITORY EVOKFID POTENTIALS IN MULTIPLE SCLEROSIS W Makuch, W Szelenberger, Z Toma每 kiewicz. M Uhrynowska FVALUATION OF COMMON CAROTID ARTER $\vec{P}$ OCCLUSION

Z Gralewski

THE EFFECTS OF INTRACEREBRAL. DOPAMINERGIC CELL TRANSPLANTATION IN RATS WITH EXPERIMENTAL PARKINSON'S DISEASI

P Koslowski 